\title{
Virtual MRTD - an indirect method to measure MRTD of thermal imagers using computer simulation
}

\author{
Krzysztof CHRZANOWSKI ${ }^{1,2,{ }^{*}, \text { NGUYEN HONG VIET }}{ }^{1}$ \\ ${ }^{1}$ Military University of Technology, Institute of Optoelectronics, \\ Kaliskiego 2, 00-908 Warsaw, Poland \\ ${ }^{2}$ INFRAMET, Bugaj 29a, Koczargi Nowe, 05-082 Stare Babice, Poland \\ *Corresponding author:kch@inframet.com
}

\begin{abstract}
Minimum resolvable temperature difference (MRTD) is considered as the most important parameter of thermal imagers. A new method of MRTD measurement without drawbacks of other methods is presented in this paper. Proposed MRTD measurement method coded as virtual MRTD is based on a three steps measurement concept using semi-automatic objective measurements and computer simulation. First, objective parameters of the tested thermal imager are measured. Second, software simulates this tested thermal imager and generates the image of 4-bar target of specified spatial frequency (size) and contrast (temperature difference). Third, a human observer analyses the images of the 4-bar target generated by the software on the screen of PC set and measures MRTD of the simulated thermal imager at specified set of spatial frequencies. The proposed method offers higher measurement speed, lower cost and typically better accuracy in comparison with the typical MRTD measurement method.
\end{abstract}

Keywords: MRTD, virtual MRTD, indirect method, computer simulation, thermal imager.

\section{Introduction}

Minimum resolvable temperature difference (MRTD) is a subjective parameter that describes the ability of the thermal imager-human system for detection of low contrast details of the observed object [ $\underline{1}-\underline{3}]$. It can be used to evaluate ranges of detection, recognition and identification of targets of interest. Therefore it is considered as the most important parameter of thermal imagers used for surveillance applications [4] and this subjective parameter is commonly measured during final product quality tests worldwide. Therefore, for decades MRTD has been a subject of interest for scientists working in field of thermal imaging. Hundreds of scientific papers have been published on measurement of MRTD, evaluation of measurement results or related subjects. It has been reported that there are several important drawbacks of classical MRTD measurement [ [ ] ]: low repeatability of measurement results due to subjective measurement, low 
speed of measurement, and phasing effects during measurements at spatial frequencies of the 4-bar target close to Nyquist frequency of the tested imager.

A series of improvements of classical MRTD measurement has been proposed [ $\underline{6}-\underline{10}]$. There are at least four competing solutions to eliminate the mentioned above limitations of classical MRTD measurement method and to improve the accuracy of range prediction: auto MRTD, dynamic MRTD, triangle orientation discrimination (TOD) and minimum temperature difference perceived (MTDP). However each of these methods has some limitations and none has been fully accepted by international community. The result is that MRTD is still measured worldwide using a classical subjective method in spite of earlier mentioned limitations [4].

A new method to measure MRTD of thermal imagers coded as virtual MRTD is presented in this paper. The proposed virtual MRTD measurement method is based on a concept of an indirect three steps measurement method using semi-automatic measurement of imager objective parameters and computer simulation of this imager. The proposed method eliminates earlier mentioned drawbacks of the classical MRTD measurement method.

\section{Classical MRTD measurement method}

MRTD is defined as a function of a minimum temperature difference between the standard 4-bar target and the uniform background required to resolve the thermal image of the bars by an observer versus spatial frequency of the target. It is a subjective parameter because the decision at what minimum temperature difference the bars of the projected image of the 4-bar target can be resolved is made by the human operator of the test system.

MRTD is typically measured using an image projector based on a reflective off-axis collimator that can project images of a set of 4-bar targets of different spatial frequency (target size) of regulated temperature difference. The observer adjusts temperature difference of an active 4-bar target to the lowest level at which 4-bar can be still resolved. The procedure is repeated for several 4-bar targets of different spatial frequency. In order to correct the so-called offset effect, the measurement is done both for positive and negative contrast. Finally, a threshold curve of spatial frequency and minimum resolvable temperature difference is built on basis of experiments (Fig. 1).

It is typically considered that the target bars are resolved if a human observer can see four separate bars at least at $50 \%$ of observation time or at least at $50 \%$ of nominal height of all four bars which are visible during observation time [4]. This measurement procedure is repeated for several observers (three observers are considered as minimum recommended number) and results are averaged.

The system for measurement of classical MRTD is typically built from a set of following blocks: off-axis reflective collimator, differential area blackbody, motorized rotary wheel, set of 4-bar targets, and a monitor (Fig. 2a). The first four blocks work as image projector. The task of the last block is to display a video image generated by a thermal imager. 


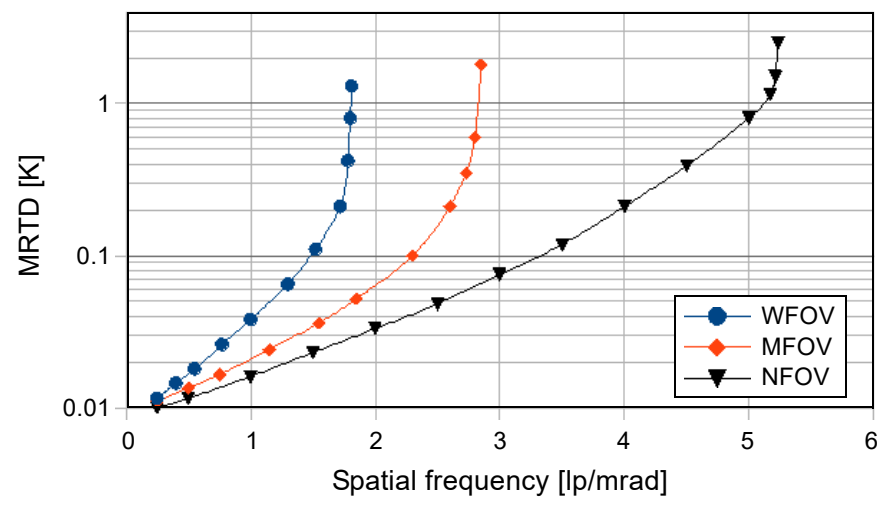

Fig. 1. MRTDs of exemplary thermal imager of three FOV.

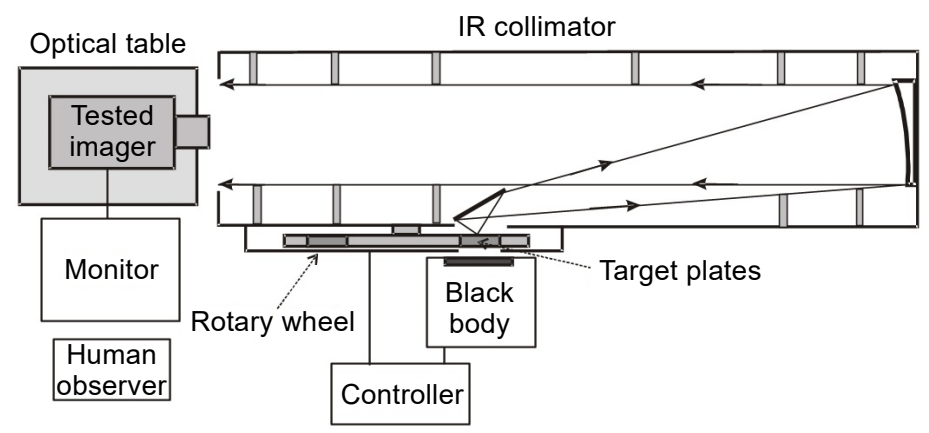

a

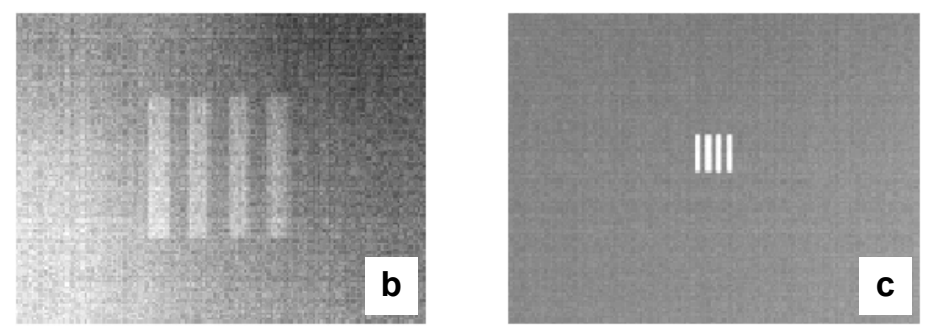

Fig. 2. Classical system for MRTD measurement of thermal imagers: block diagram (a), projected images of two 4-bar targets $(\mathbf{b}, \mathbf{c})$.

This classical MRTD measurement method is characterized by several drawbacks:

1) subjective measurement,

2) slow speed of measurement,

3) high cost of a large set of 4-bar targets,

4) technical problems to manufacture high frequency 4-bar targets,

5) phasing effect close to Nyquist frequency of the tested imager that limits measurement accuracy.

Subjective character of MRTD measurement using a classical measurement method has some disadvantages and advantages. The disadvantages, because the results of 
MRTD measurement vary depending on observers. In detail, even the same observer can generate slightly different MRTD measurement results if tests are repeated. However, these drawbacks are reduced by using several observers for MRTD measurement and by averaging the measurement results. The advantage is the fact that surveillance thermal imagers that dominate the market are intended to be used by humans and therefore tests performed by humans increase test realism.

MRTD is not a single value parameter but a function. Therefore, if MRTD function is to be determined with good accuracy then it should be measured in a range from low frequency to high frequency slightly over Nyquist frequency. Practically it means that MRTD is to be measured at least five measurement points (values of spatial frequency). The measurements are to be done at both positive and negative contrast. Next, the measurements are often done for both vertical and horizontal orientation of the 4-bar target. Finally, the measurements are to be repeated by at least three observers. The result is that if MTRD of a long range thermal imager with three FOV is to be accurately measured (at least five points at every FOV, both positive and negative contrast, both vertical and horizontal orientation, three observers) then tested MRTD is to be measured at least 180 measurement points.

Time needed to change and to stabilize temperature of the blackbody from one temperature to another is typically at least 30 seconds. At the same time, typically a dozen of such changes is needed to find proper temperature difference for a single spatial frequency. Such a situation makes MRTD measurement very slow. Half a day or more is often needed to test a single multi-FOV thermal imager.

As it was earlier mentioned, MRTD is to be measured at at least 5 spatial frequencies. The range about the Nyquist frequency is critical. However, the Nyquist frequency of different thermal imagers varies significantly. Practically it means that it is necessary to have dozens of 4-bar targets to be able to do MRTD measurement of every thermal imager offered on the market. This high number of 4-bar targets needed for MRTD measurement generates significant cost. Lack of proper 4-bar targets is a common problem for many test teams because they do not have suitable targets that should be tested just now.

In addition, the measurement of MRTD of some modern thermal imagers requires using of 4-bar targets of extremely high spatial frequency in $\mathrm{lp} / \mathrm{mm}$ up to $25 \mathrm{lp} / \mathrm{mm}$ or more. Practically it means that 4-bar targets having bars of width as small as $0.02 \mathrm{~mm}$ or less are needed. Manufacturing of any 4-bar having bars below $0.1 \mathrm{~mm}$ presents a technical challenge due to problems of how to achieve high accuracy of cutting rectangle holes in a thin metal sheet, to create a high emissivity coating and to keep high thermal stability of the target plate.

The phasing effect is a phenomenon when the ability to resolve bars of 4-bar target of spatial frequency close to Nyquist frequency depends on its angular position. Slight movement of an angular position can drastically change MRTD measurement results of undersampled thermal imagers (typical situation for great majority of thermal imagers).

The earlier presented limitations of a classical MRTD measurement method are known almost since the beginning of thermal imaging metrology. Therefore it is not 
surprising that at least from 1980s there are many scientific projects to develop solutions to overcome these limitations of classical MRTD.

\section{Solutions to remove limitations of classical MRTD}

These solutions proposed in different literature sources to overcome limitations of classical MRTD can be divided basically into two groups:

1) Non-classical MRTD measurement methods,

2) New parameters that could replace the classical MRTD.

\subsection{Non-classical MRTD measurement methods}

There are at least two widely used non-classical methods of measuring MRTD of thermal imagers that are known under following names: auto MRTD [ [ $]$ and dynamic MRTD (DMRTD) [ㅁ].

Auto MRTD is a fully objective method to measure MRTD that proposes the calculation of MRTD on the basis of measured two objective parameters NETD and MTF.

In detail, auto MRTD is to be measured using a five steps algorithm:

1) Measure the classical subjective MRTD, and two objective parameters (NETD - noise equivalent temperature difference, and MTF - modulation transfer function) of a dozen of one type of thermal imagers.

2) Calculate the average classical MRTD, NETD, MTF for tested sample cameras.

3) Calculate the coefficient function $K(v)$ as

$$
K(v)=\frac{\operatorname{MRTD}(v) \operatorname{MTF}(v)}{\operatorname{NETD}}
$$

4) Measure MTF and NETD of new thermal imagers of unknown MRTD.

5) Calculate auto MRTD of new thermal imagers using the following formula:

$$
\operatorname{MRTD}_{\text {auto }}(v)=\frac{K(v) \text { NETD }}{\operatorname{MTF}(v)}
$$

Measurement of both MTF and NETD is fast and semi-automatic. Therefore the measurement time needed to determine the objective $\mathrm{MRTD}_{\text {auto }}$ is much shorter than the time needed to measure the classical subjective MRTD. However, because the determination of $K(v)$ requires preliminary testing of a sample of thermal imagers, $\mathrm{MRTD}_{\text {auto }}$ can be used only in case of large production lines. Other drawbacks of the classical MRTD like costs of 4-bar targets, problems to manufacture small 4-bar targets, phasing effect close to Nyquist frequency are still valid.

The latter drawback of the classical MRTD is overcome by the second non-classical MRTD measurement method known as dynamic MRTD (DMRTD). This method is based on a concept to use dynamic 4-bar targets during MRTD tests. Practically it means that a 4-bar target is moving slowly in direction perpendicular to the bars with 
a constant speed in FOV of the tested thermal imager. The problems of phase optimization and beat frequency disruption (phasing effects) of the 4-bar patterns can be fully eliminated. However, the integration time of the IR FPA, the human visual system and the imager MTF influence the results of the DMRTD test. Therefore an optimal speed for target motion varies from case to case and this fact is a significant limitation of the DMRTD method. A necessity to use sophisticated mechanics to ensure the precision movement of a 4-bar target (or collimator) is the second, even more important drawback of the DMRTD method.

\subsection{New parameters that could replace the classical MRTD}

There are two parameters that have high potential to replace the classical MRTD in future: triangle orientation discrimination (TOD) threshold [ [ -9 ] , and minimum temperature difference perceived (MTDP) [10].

TOD methodology is an alternative approach to characterize performance of thermal imagers using a non-periodic target (equilateral triangle) instead of a classical 4-bar target. In addition, three stages of measurements need to be followed to obtain TOD curve:

1) A target of an equilateral triangle of a specified edge size is projected at random of four possible orientations (up, down, left and right). The contrast of a target is to be varied from high to low. For each measuring point, a human observer must indicate the orientation of the triangle she/he sees several times, even if she/he is not sure what is a proper orientation. Each response is scored as right or wrong. Percent of correct responses for a given size and contrast can be changed from $25 \%$ (pure guesswork) to $100 \%$ (full clarity).

2) For each specified size of an equilateral triangle, a curve of a right definition of orientation is built. Contrast, where the probability of a right definition of orientation is $75 \%$ will be taken to account.

3) TOD curve is graphically built as a function of a triangle size versus contrast necessary for an observer to accurately $(75 \%)$ resolve it.

Spatial frequency of the triangle is converted from an angular size by taking a square root of triangle area into milliradian. The concept and procedure of TOD measurement are clear and objective. TOD method has a number of advantages over the classical MRTD but also has disadvantages. The first limitation of the TOD method is to develop the equipment that enables generation of four random directions of an equilateral triangle target. The second is a difficult mathematical conversion from TOD to MRTD that is needed to calculate ranges of effective surveillance of the tested thermal imager. The last disadvantage is a high cost of a set of small triangle targets, the same drawback as of the classical method.

MTDP can be considered as the evolution of the classical MRTD concept [11]. In MTDP measurement the minimum temperature difference can be accounted even when only three or two bars are resolved by an observer, with the 4-bar target at the optimum phase position. Therefore it is possible to measure MTDP at frequencies much over 
Nyquist frequency [12] when such measurements are not possible in the case of classical MRTD. The detailed description of MTDP methodology and full procedure can be found in Ref. [10].

\subsection{Emerging solutions}

Besides four competing methods reviewed above, there are at least two new emerging solutions that also compete to replace the subjective and time consuming classical MRTD.

Reference [13] proposes to use machine learning in MRTD measurement. In detail, the back-propagation algorithm is used in training feed-forward neural networks in measurement process. The results show that the proposed method can meet the MRTD measurement requirements.

Reference [14] presents the results of the experiment with a computer program to analyse the images of 4-bar targets generated by tested thermal imagers at a given objective threshold (signal-to-noise). In detail, this paper shows that by using a signal analysis algorithm to analyse images of 4-bar target it is possible to obtain machine generated MRTD curves. It was shown that if the required signal-to-noise ratio is optimized then the machine-based MRTDs can be very similar to the ones obtained with the help of the human observer.

\subsection{Review of solutions to overcome classical MRTD}

All these four competitive techniques shortly reviewed in previous sections have been developed to replace classical MRTD due to the drawbacks of this classical method. However, all these new methods have some drawbacks, too. Based on the above analysis, a list of drawbacks of MRTD measurements is created for clear comparison (see the Table).

The analysis of data in the Table shows that each of known methods to measure MRTD has some limitations and a new method is needed that fully eliminates drawbacks of the classical method.

T a b l e. Drawbacks of MRTD measurement methods.

\begin{tabular}{ll}
\hline MRTD measurement method & Drawbacks \\
\hline \multirow{2}{*}{ Classical subjective MRTD } & Subjective character of measurement. \\
& $\begin{array}{l}\text { Low measurement speed. } \\
\text { High price of large set to 4-bar targets needed for accurate tests. } \\
\text { Phasing effect close to and over Nyquist frequency. }\end{array}$ \\
\hline Auto MRTD & Low accuracy when testing small quantities imagers. \\
\hline Dynamic MRTD & Technical problems to achieve proper speed of angular rotation. \\
\hline \multirow{2}{*}{ TOD } & Test target totally different from classical 4-bar shape. \\
\hline MTDP & Difficult conversion from TOD to MRTD. \\
\hline & Lack of support from standards. \\
& Users has problems to interpret measurement data. \\
\hline
\end{tabular}




\section{Concept of virtual MRTD measurement method}

Virtual MRTD is a method to measure MRTD based on an idea of using computer simulation to create a virtual copy of a tested imager and to do later classical MRTD measurement by testing this virtual imager. In detail, virtual MRTD is based on a concept to measure MRTD of thermal imagers using a three steps measurement algorithm (Fig. 3):

1) Measurement of objective parameters (MTF, 3D-noise components, FOV) of tested thermal imager.

2) Computer simulation of tested thermal imager during MRTD tests on the basis of measured data in previous step.

3) Analysis of images of 4-bar target of variable spatial frequency and variable temperature difference by a human observer and determination of MRTD of this virtual thermal imager.

The system needed for testing thermal imagers using virtual MRTD method shown in Fig. 4 can be built by several modifications of the system for measurement of classical MRTD shown in Fig. 2a. These modifications are:

1) Frame grabber needed for acquisition of video images generated by the tested thermal imager.

2) Additional set of targets (edge/slit target needed for MTF measurement, multi -point cross target for FOV measurement, optional square target needed for measurement of noise/response parameters (3D-noise model)).

3) PC set as a main computing unit.

4) $\mathrm{PC}$ monitor to display images captured by a frame grabber.

5) Software to control a blackbody and rotary wheel.

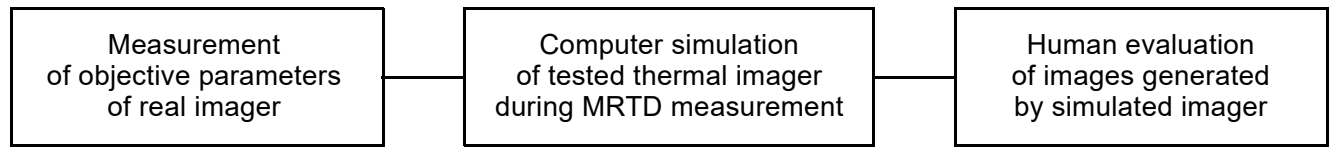

Fig. 3. Graphical concept of virtual MRTD measurement method.

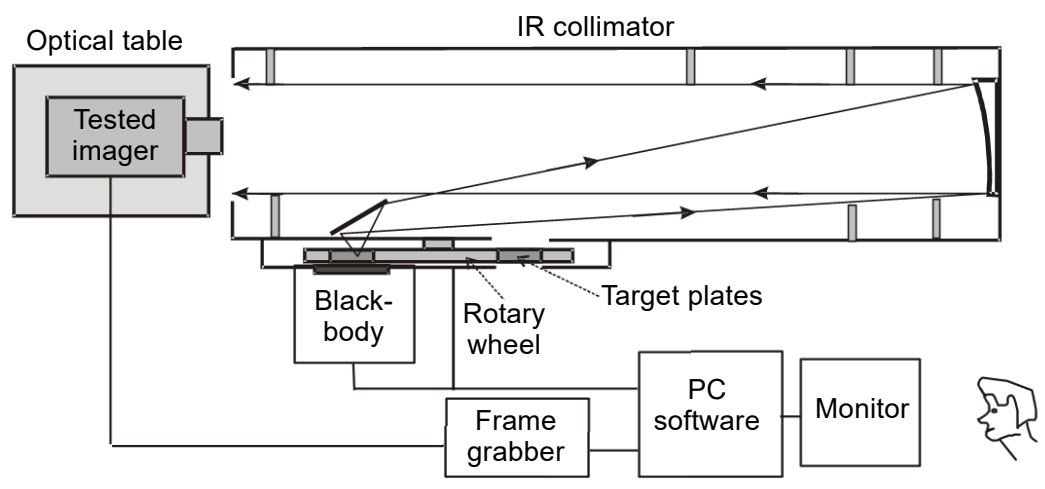

Fig. 4. Block diagram of system for testing thermal imagers using virtual MRTD method. 
6) Software for acquisition, image processing and measurement of MTF, 3D-noise parameters, FOV.

7) Software to simulate the tested imager and do virtual MRTD tests.

The system for testing thermal imagers using a virtual MRTD method can be divided into two subsystems:

1) System for measurement of both classical MRTD and main objective parameters (MTF, 3D-noise, FOV) built as a system for measurement of the classical MRTD with additional blocks Nos. 1-7.

2) Computer program to simulate the tested thermal imager during MRTD measurement process.

The systems for measurement of classical MRTD, MTF, 3D-noise, FOV can be easily obtained at the international market as such systems are offered by many manufacturers [15-18]. Computer program to simulate the tested thermal imager during MRTD measurement process is the new crucial block of a system for testing thermal imagers using a virtual MRTD method and need to be discussed in detail.

\section{Computer program to simulate thermal imagers}

A computer program coded Dubterm that converts typical PC into a virtual thermal imager during MRTD measurement process has been developed. The program enables the simulation of a tested thermal imager during MRTD measurement process. In detail, the program on the basis of measured objective parameters (MTF, 3D-noise, FOV) treated as an input data generates on PC screen an image of a 4-bar target merged with noise background that is almost identical as the image generated by a real thermal imager during such tests. The size and contrast of a generated 4-bar target can be regulated like during the real life test. A user can determine what is a minimal temperature difference when he can still resolve four bars of a simulated 4-bar target.

The program consists of five basic modules: the targets editor module, the tests conditions module, the imager simulation module, the visualization module, and the measurement process module.

The targets editor module enables creation and editing of the parameters of a test target: number of 4-bar targets to be used in measurement process, target dimensions (bar width of the target), target position in FOV of the tested imager (centre position or random position), and optional movement speed of a test target (potential simulation of dynamic MRTD).

The test conditions module enables control of parameters of the collimator (focal length and transmittance), ambient temperature and conditions for MRTD measurement process (number of observers, type of MRTD tests (both positive and negative contrast or only positive contrast)). In addition, the module calculates radiant signals emitted by the target and the background that come to the imager input and create the input scene.

The imager simulation module is the heart of the Dubterm image generator. This module enables the import of a series of measured objective parameters of the simu- 
lated thermal camera: MTF, 3D-noise parameters, and FOV. The user is expected also to specify a spectral band of this tested imager. On the basis of these imported camera parameters and the data from the previously discussed modules the imager module calculates the final output image of the 4-bar target.

The visualization module creates the final output thermal image for the current camera settings like: the mode of the field of view (narrow, wide), brightness, contrast, polarity, and digital zoom. The users can use a manual mode when they can control manually all these settings or automatic mode when they program and optimize the brightness level and contrast.

The measurement process module enables to carry out the classical MRTD measurement procedure (changing active target, regulation of temperature, recording of values of minimal temperature differences when an observer can resolve four bars of the target, presentation of MRTD data in the form of a table, and graphical presentation of MRTD data) using a virtual thermal imager simulated on monitor.

Dubterm is a computer program written in $\mathrm{C}++$ using OpenGL graphic library that can work in the Windows $7 / 10$ operating systems. It is fine, a user friendly computer program but the crux of this program is not programming but mathematical modelling that makes possible the simulation of thermal imagers.

\section{Mathematical modelling of thermal imagers}

Mathematical model of thermal imagers during measurement of MRTD used by Dubterm computer program is based on the following assumptions:

1) Thermal imager can be considered as an image processing system that converts a perfect analog image of the target of interest (4-bar target) into a non-perfect digital copy of the input image.

2) Non-perfection of the output image is generated due to three effects: blurring, noisiness, and digitization.

3) The image blurring can be mathematically described using a theory of linear filtration on the basis of a measured MTF function of the tested imager.

4) Fast Fourier transform algorithms can be used to accurately model the blurring of an input analog image into an output digital image on condition that the calculations shall be carried out at an image resolution at least 16 times higher than the resolution of the output image.

5) The image noisiness can be mathematically described using 3D-noise model parameters of the tested imager.

6) Digitization effect can be described by the resolution of IR FPA sensor used by the tested thermal imager.

7) Both MTF and 3D-noise parameters do not vary in time and do not depend on imager settings. Practically it means that the tested thermal imager can be fully characterized by one time measurement.

The first assumption is almost fully fulfilled. There is no logical arguments why the thermal imager cannot be considered as an image processing system that degrades 
the perfect input analog image. The questionable is only the assumption that the output image is digital in situation when the majority of thermal imagers generate an output video image in analog video standards. However, the fraction of fully digital thermal imagers is quickly rising. In addition, the analog video image can be converted into the digital video image using frame grabbers.

The second assumption appears also easy to be accepted. Both noise and blurring can have a very broad meaning. It is also clear that image digitization can be considered as a form of image degradation.

The third assumptions can be treated as questionable. Modern thermal imagers are electronic devices that are partially non-linear and use advanced image processing that can limit the accuracy of a linear filtering theory used to model the effect of image blurring. In addition, the assumption concerning the use of MTF as a basic input parameter means that it was assumed that there is a symmetric response of a thermal imager to input point sources. However, for small temperature differences thermal imagers are proved to be quasi-linear. Next, the deviations from symmetric response are typically small.

Fast Fourier transform (FFT) is a convenient tool for fast image processing of digital images. However, initial experiments carried out by the authors have shown that accurate blurring of an input analog image into an output digital image using FFT tools is not possible if calculations are carried out at image resolution equal to IR FPA resolution. These experiments have shown that high accuracy of blurring process can be achieved if calculations are carried out at image resolution at least 16 times higher comparing to IR FPA resolution.

The fifth assumption appears to be relatively sound. 3D-noise is a complex model when noise is characterized by a set of eight parameters [19]. All these parameters describe in detail different types of noise present in a video image generated by thermal imagers. When combined together these eight noise components give very realistic description of noisiness of a video image.

The sixth assumption is the easiest to accept. It is obvious that a digitization effect can be described by the resolution of IR FPA sensor used by the tested thermal imager. However, it should be kept in mind that in the case of thermal imagers with an analog video output, the digital image of resolution equal to resolution of IR FPA is converted to the analog video image of PAL/NTSC format of slightly higher image resolution.

The seventh assumption is never fully fulfilled. 3D-noise model parameters always vary with time. The same occurs but to a smaller degree with MTF. In addition, both parameters depend slightly on imager settings like gain and brightness. However, both the variations and dependences are not typically significant and this assumption can be considered as justified.

After discussing the assumptions, let us present a mathematical algorithm. From mathematical point of view, Dubterm works according to a following algorithm:

1) Import of raw video image data used to measure objective parameters (MTF, 3D-noise, FOV) captured at standard image resolution/dynamic (image resolution of IR FPA sensor and image of standard 8 bit dynamic). 
2) Conversion of the captured raw video image to high resolution/high dynamic image. The latter term means the image of resolution 16 times higher over standard sensor resolution and image of 16 bit dynamic. Interpolation algorithms are used to increase the image resolution/dynamic of captured raw video image.

3) Determination of MTF, 3D-noise, FOV of tested thermal imager.

4) Creation of analytical model of input scene for specified test conditions (size of active 4-bar target, collimator type, ambient temperature).

5) Conversion of analog input scene to high resolution/dynamic input image.

6) Operation of image blurring of the input high resolution/dynamic image.

7) Operation of degradation of noise-free blurred high resolution/dynamic video image calculated in previous step by adding all components of 3D noise.

8) Conversion of high resolution/dynamic blurred, noisy output image to standard resolution/dynamic image to be analyzed by an observer.

9) Graphical presentation of video image calculated in previous step on PC screen. User can regulate both temperature difference of the simulated target and different imager settings (contrast, brightness, zoom, FOV).

10) Determination by the user of minimal temperature difference between target bars and background when the user can resolve all four bars.

11) Repeating of the steps $4-10$ for different targets and calculation of virtual MRTD.

The steps 6-8 are of critical importance and shall be described in detail.

Operation of image blurring of the input high resolution high dynamic image is done in three stages (Fig. 5). First, Fourier transform is performed on the input scene image (high resolution/dynamic image). Second, a multiplication of Fourier transform of the input scene and MTF of the simulated thermal imager is done. Third, an inverse Fourier transform from result of the previous step is performed. The blurring operation is done

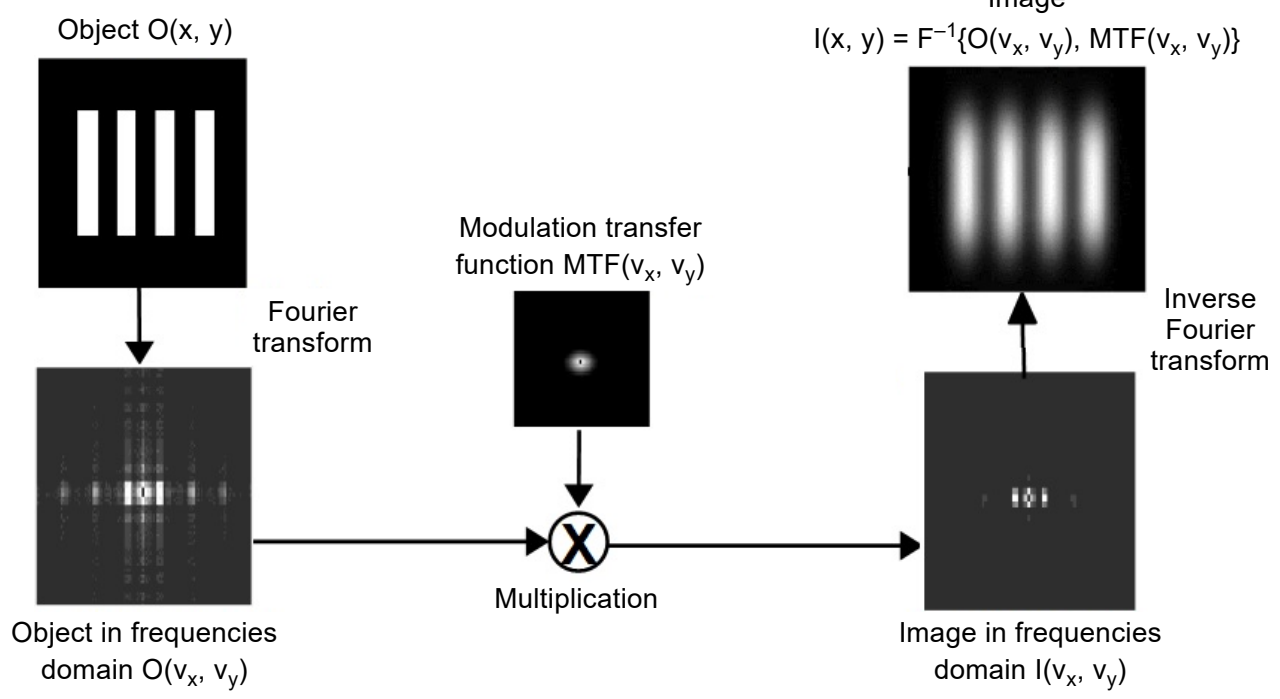

Fig. 5. Graphical mathematical model used for blurring operation. 
for two dimensional blurring based single-orientation measured MTF (assumption that horizontal MTF equals to vertical MTF) or first horizontal blurring and later vertical blurring if data on measured MTF of tested imager at two directions are available. Fast Fourier transform is used for both stages to speed up calculations.

Operation of the degradation of noise-free blurred high resolution/dynamic video images is done by adding a series of noise components of 3D-noise model. In detail, the output signal for each pixel of the analysed output image is calculated as a sum of input signals of the current pixel of interest and a series of noise random variables that represent different noise components:

$$
\begin{aligned}
U(t, v, h)= & I(t, v, h)+N\left(0, \sigma_{\mathrm{t}}\right)+N\left(0, \sigma_{\mathrm{v}}\right)+N\left(0, \sigma_{\mathrm{h}}\right)+N\left(0, \sigma_{\mathrm{tv}}\right) \\
& +N\left(0, \sigma_{\mathrm{vh}}\right)+N\left(0, \sigma_{\mathrm{tvh}}\right)+N\left(0, \sigma_{\mathrm{th}}\right)
\end{aligned}
$$

where $t$ is the time coordinate; $v$ and $h$ are spatial coordinates of the pixel of interest; $U(t, v, h)$ is the output signal at the coordinates $t, v, h ; I(v, h)$ is the input signal for the pixel of coordinates $v$ and $h$; $N$ is the noise random variable of expected values equal to zero and standard deviation $\sigma$, and $\sigma_{\mathrm{t}}, \sigma_{\mathrm{v}}, \sigma_{\mathrm{h}}, \sigma_{\mathrm{tv}}, \sigma_{\mathrm{th}}, \sigma_{\mathrm{vh}}, \sigma_{\mathrm{tvh}}$ are components of 3D-noise model.

The conversion of an output high resolution blurred, noisy output image $U(t, v, h)$ to standard resolution/dynamic video image is a simple scaling down operation of a high resolution/dynamic bitmap to a lower resolution/dynamic bitmap. It is typically a conversion of 16 bit bitmap of resolution $10240 \times 7680$ pixels to 8 bit bitmap of resolution $640 \times 480$.

\section{Experimental verification of virtual MRTD method}

The authors carried out the experimental verification of a hypothesis proposed in this paper by making MRTD measurement of a series of thermal imagers using two measurement methods:

1) Classical MRTD measurement method;

2) Virtual MRTD measurement method.

The tests have been carried out using two upgraded commercially available test systems coded as TAIM (Fig. 6a) and MS300 (Fig. 6b) [15]. The TAIM/MS300 are commercial codes of typical test systems (collimator, blackbody, rotary wheel, set of targets, PC set, frame grabber, control software, and test software). The word upgraded means that earlier discussed computer program Dubterm has been installed on the PC of the test TAIM/MS300 systems. Two test systems have been used to fit better to tested two groups of thermal imagers: short range imagers and large ultra long range imagers.

Over a dozen of thermal imagers has been tested. Here we present only results obtained for only two thermal imagers that can be considered as representatives from two main groups of surveillance thermal imagers offered on the international market:

1) Short/medium range portable non-cooled LWIR thermal imager,

2) Long range stationary/mobile cooled MWIR thermal imager. 

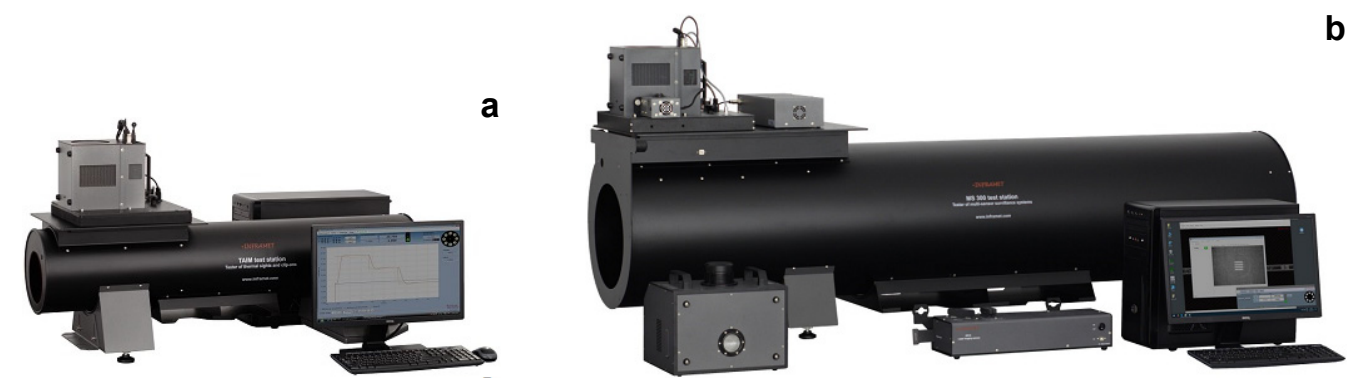

Fig. 6. Photos TAIM (a), and MS300 (b) test systems.

Non-cooled LWIR thermal camera with LWIR objective of $100 \mathrm{~mm}$ focal length and of $6.5^{\circ} \times 4.9^{\circ} \mathrm{FOV}$ from Etronika Ltd is a representative of short/medium range non-cooled LWIR thermal imagers.

Cooled MWIR imager with zoom optics at $825 \mathrm{~mm}$ focal length and $0.67^{\circ} \times 0.53^{\circ}$ FOV coded as HeatSeekIR 4H from Optix Co Bulgaria is a representative of the long range cooled MWIR thermal imagers.

Measurement results of MRTD of these two exemplary thermal imagers obtained using two measurement methods are shown in Fig. 7.

The measurement results similar to the data shown in Fig. 7 have been obtained in case of other tested thermal imagers. Therefore the following conclusions can be formulated on the basis of obtained experiments:

1) There is a good agreement between the classical MRTD measurement method and the virtual MRTD measurement method. Both classical MRTD and virtual MRTD show the same trends.

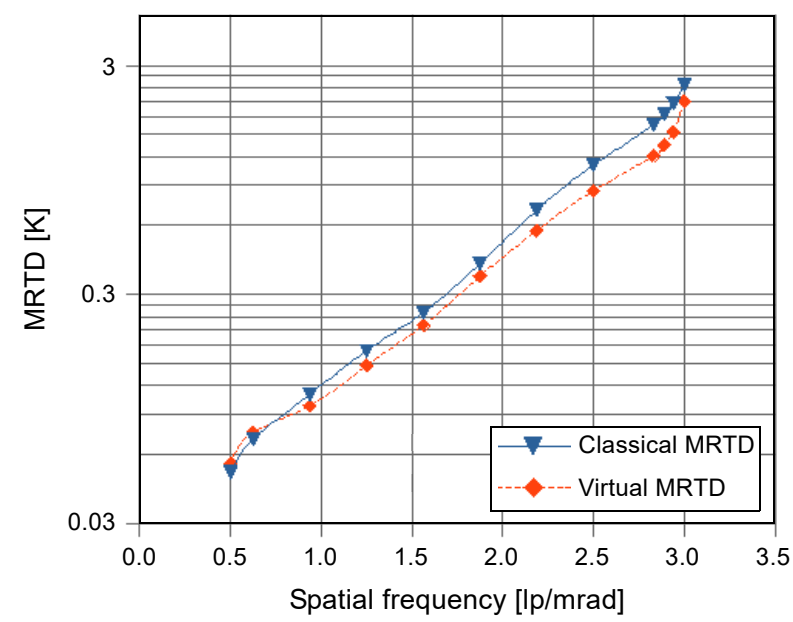

a

Fig. 7. MRTDs of the exemplary non-cooled LWIR (a), and cooled MWIR (b) thermal imagers. 


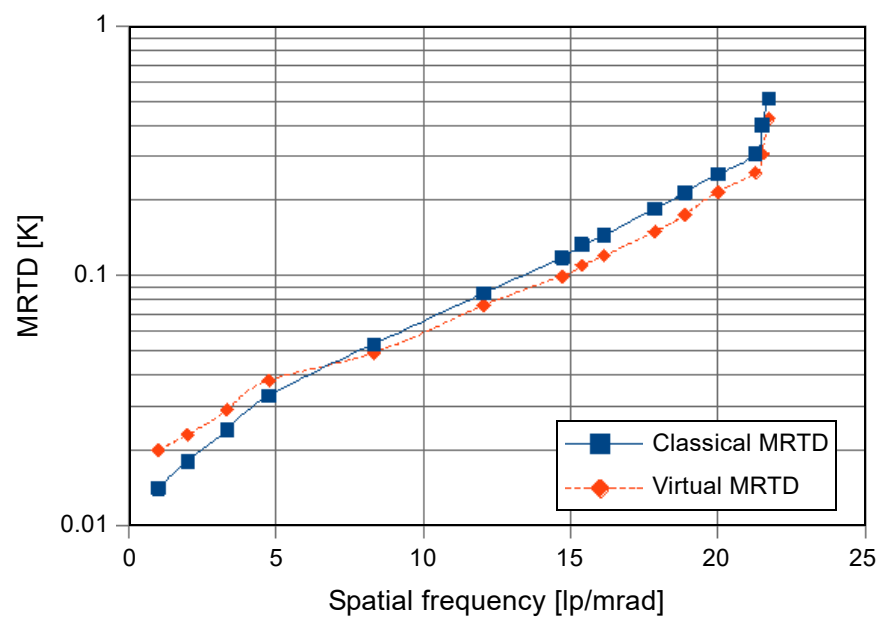

b

Fig. 7. Continued.

2) The highest relative differences are recorded at a low spatial frequency range. The authors cannot fully explain the reason for such a situation. However, this range is not important in main application of MRTD: calculation of recognition ranges according to NATO standard (high and medium ranges are used).

3) The recorded differences between the classical MRTD and the virtual MRTD are noticeable but still are surprisingly low if we consider the differences at a level as high as $50 \%$ which are often cited between two laboratories using the same classical MRTD measurement method [2]].

\section{Discussion}

The results of experiments presented in previous section show that if the classical MRTD and virtual MRTD are carried out by the same test team then the differences are at a relatively low and acceptable level. Practically it means that virtual MRTD can be treated as a potential near perfect MRTD measurement method due to a series of advantages.

First, measurement time of MRTD using virtual MRTD method is many times shorter comparing to the classical measurement method. The main gain comes from the fact that a user can regulate temperature of virtual blackbody without any noticeable delay while in the case of real blackbody it is necessary to wait up to one minute to have the blackbody stabilized at a single chosen temperature and experiments are to be carried out sometimes for hundreds of temperature points.

Second, cost of testing a series of different thermal imagers using the virtual MRTD method is much lower comparing to the cost of such tests using a classical MRTD method. Time factor was already mentioned. The second factor is a reduced number of re- 
quired targets. In case of virtual MRTD only two targets (the edge target needed for MTF measurement and dot cross target for FOV measurement) are needed when many dozens of 4-bar targets are needed in case of classical MRTD method.

Big number of 4-bar targets originates from the fact that there are myriads of types of thermal imagers at an international market having Nyquist frequency that vary significantly from about $0.03 \mathrm{lp} / \mathrm{mrad}$ in case of imagers with extremely wide FOV to about $40 \mathrm{lp} / \mathrm{mrad}$ in case of imagers with extremely narrow FOV. At the same time, it is critical to measure MRTD at frequencies close to Nyquist frequency because measurements at this spatial frequency range are needed later for accurate calculation of ranges of detection, recognition and identification of targets of interest using the tested imager.

Third, phasing effect close to Nyquist frequency of the tested imager can be eliminated when testing using virtual MRTD method by activating angular movement of the virtual target at speed about $1 / 4$ pixel per frame. The same effect can be achieved also in the case of real systems by moving the bar pattern relative to the sampling lattice with optimum speed (about $1 / 4$ pixel per frame). However, in the case of a real test system such solution is a technical challenge due to the necessity to develop a precision mechanical stage that generates a significant price increase of the required test system.

Fourth, virtual MRTD method gives a test team the ability to keep testing conditions unchanged at any time. That means that testing conditions are the same for each observer in the test team. This feature of virtual MRTD method will help to increase the accuracy and repeatability of MRTD measurement. The importance of this feature is known for all experienced MRTD test teams. It is a common situation that tests of the same thermal imager at some time intervals generate often slightly different results due to minimally changed ambient temperature, imager settings or other factors.

Fifth, virtual MRTD method is a near perfect solution to solve problems of measurement of MRTD of thermal imagers to be used at extreme temperatures (ambient temperatures below $-20^{\circ} \mathrm{C}$ or over $+40^{\circ} \mathrm{C}$ ). The simplest solution for such tests is to insert both the test systems, tested imager and human crew to a big temperature chamber. However, very few humans can withstand the work for several hours in temperature chambers in such extreme temperatures. Next, the expensive big chamber is out of any other use for a long time. In case of virtual MRTD method only short duration measurement of objective parameters of tested thermal imager is needed. Next, a human operator can be outside the chamber if needed. Virtual MRTD tests can be later done at comfortable laboratory conditions.

The proposed virtual MRTD method of MRTD measurement is based on a concept that differs very significantly from any other known methods: auto MRTD, dynamic MRTD, TOD, and MTDP. The closest is auto MRTD. Virtual MRTD like auto MRTD requires to measure some objective parameters of a thermal imager but the measurement is done in a totally different way. In case of auto MRTD a simple mathematical formula must be the same for a long batch of tested imagers. In case of virtual MRTD the typical measurement using a human operator is imitated using a computer simulator employing a sophisticated mathematical model that varies from case to case. There- 
fore, a possibility that a poorly working thermal imager can pass virtual MRTD (like it is common in auto MRTD) is near zero.

There are also some limitations of this proposed MRTD measurement method.

First, virtual MRTD cannot be used to measure MRTD of analog thermal imagers that present video image on analog video monitors or for testing a direct view thermal imager that do not have outputs of an electronic video image. Virtual MRTD can be used only when a digital video image from tested imager can be captured (digital thermal imagers or analog imagers but integrated with frame grabbers). However, it should be emphasized that a fraction of digital thermal imagers on international market is quickly rising and such imagers will soon be market majority.

Second, virtual MRTD can be used for accurate MRTD measurement only for thermal imagers having a quasi-symmetrical point spread function PSF (symmetrical image blur of a point source) because the mathematical simulation model of virtual MRTD assumes that the imager optical transfer function OTF equals the modulation transfer function MTF neglecting phase transfer function PTF contribution. However, the measurement of MRTD is typically done using a target located in the centre of an imager FOV where the point spread function PSF is typically quasi-symmetrical and neglecting PSF does not generate the noticeable loss of realism of an output image.

The authors have made an assumption that OTF equals to MTF due to a market reality when typical systems for testing thermal imagers do measure MTF but do not measure PTF. However, the authors plan in near future to change algorithm, modify software to include PTF influence on output image and to eliminate this assumption.

\section{Conclusions}

The proposed method of MRTD measurement of a thermal imager coded as a virtual MRTD method offers a series of advantages over classical MRTD, modified measurement methods (dynamic MRTD, auto MRTD) or different known solutions to replace classical MRTD like TOD or MTDP. In detail, virtual MRTD offers higher measurement speed, lower cost and typically better accuracy in comparison with classical MRTD or equivalent measurement methods. Therefore it can be expected that in future a significant fraction of MRTD measurement of thermal imagers shall be done using virtual MRTD method.

Acknowledgements - Research presented in this paper was funded by a grant from the National Center for Research and Development of Poland No. MAZOWSZE/0043/19.

\section{References}

[1] NATO, Definition of Nominal Static Range Performance for Thermal Imaging Systems, STANAG 4347, 1995.

[2] Holst G.C., Testing and Evaluation of Infrared Imaging Systems, JCD Publishing Co., 1993.

[3] Chrzanowski K., Testing thermal imagers, Military University of Technology, 2010.

[4] Perić D., Livada B., MRTD Measurements Role in Thermal Imager Quality Assessment, 2019. 
[5] De Jong A.N., Bakker S.J.M., Fast and objective MRTD measurement, Proceedings of SPIE 916, 1988, pp. 127-143, DOI: $10.1117 / 12.945571$.

[6] Webi C.M., Halford C.E., Dynamic minimum resolvable temperature testing for staring array imagers, Optical Engineering 38(5), 1999, pp. 845-851, DOI: 10.1117/1.602281.

[7] Bijl P., Valeton J., Triangle orientation discrimination: the alternative to minimum resolvable temperature difference and minimum resolvable contrast, Optical Engineering 37(7), 1998, pp. 1976 -1983, DOI: $10.1117 / 1.601904$.

[8] McHugh S.W., IRwin A., VALETOn J.M., BiJl P., TOD test method for characterizing electro-optical system performance, Proceedings of SPIE 4372, 2001, pp. 39-45, DOI: 10.1117/12.439159.

[9] BiJl P., VAlETon J.M.,Guidelines for accurate TOD measurement, Proceedings of SPIE 3701, 1999, pp. 14-25, DOI: $10.1117 / 12.352986$.

[10] Wittenstein W., Minimum temperature difference perceived - a new approach to assess undersampled thermal imagers, Optical Engineering 38(5), 1999, pp. 773-781, DOI: 10.1117/1.602265.

[11] NATO, Experimental Assessment Parameters and Procedures for Characterisation of Advanced Thermal Imagers, North Atlantic Treaty Organisation, Research and Technology Organization, Neuilly-sur-Seine Cedex, France, 2003.

[12] WitTEnSteIn W., Thermal range model TRM3, Proceedings of SPIE 3436, 1998, pp. 413-424, DOI: $10.1117 / 12.328038$.

[13] XU L., Li Q., Lu Y., Method of object MRTD-testing for thermal infrared imager, Proceedings of SPIE 10815, 2018, article 108151F, DOI: 10.1117/12.2502131.

[14] Van Rheenen A.D., Taule P., Thomassen J.B., Madsen E.B., MRTD: man versus machine, Proceedings of SPIE 10625, 2018, article 106250N, DOI: $10.1117 / 12.2304581$.

[15] www.inframet.com (accessed April 23, 2020).

[16] www.hgh-infrared.com (accessed April 23, 2020).

[17] www.ci-systems.com (accessed April 23, 2020).

[18] www.sbir.com (accessed April 23, 2020).

[19] O'Shea P., Sousk S., Practical issues with 3D noise measurements and application to modern infrared sensors, Proceedings of SPIE 5784, 2005, pp. 262-271, DOI: 10.1117/12.604588.

Received April 23, 2020

in revised form June 12, 2020 